\title{
Informant questionnaire on cognitive decline in the elderly (IQCODE) for assessing the severity of dementia in patients with Alzheimer's disease
}

\author{
Yunlong Ding ${ }^{1 \dagger}$, Jiali Niư ${ }^{2 \dagger}$, Yanrong Zhang ${ }^{1}$, Wenpeng Liu', Yan Zhou ${ }^{3}$, Can Wei ${ }^{1}$ and Yan Liu $^{1 *}$
}

\begin{abstract}
Background: The Informant Questionnaire on Cognitive Decline in the Elderly (IQCODE) is widely used as a complementary screening tool for dementia. However, there are few studies concerning the efficacy of the IQCODE for assessing the severity of cognitive impairments in patients with Alzheimer's disease (AD). We aimed to evaluate the efficacy of the IQCODE for assessing the severity of dementia in patients with AD.

Methods: According to the clinical dementia rating (CDR), 394 patients with AD were enrolled and classified into three groups: mild, moderate and severe groups. The IQCODE scores of each group were determined by interviewing the informants with the short version of the 16-item IQCODE. The correlations of the IQCODE score with the Mini-Mental State Examination (MMSE), the Mattis Dementia Rating Scale (DRS) and the Alzheimer's Disease Assessment Scale-Cognitive Subscale (ADAS-Cog) were analysed. Statistical analyses were conducted to examine the differences in the IQCODE scores among the three groups.

Results: The validity coefficients of the IQCODE with the MMSE, DRS and ADAS-Cog were $-0.528,-0.436$, and 0.477 , respectively. The sensitivity was $66.1 \%$, and the specificity was $59.8 \%$ when using a cut-off score of 65 to discriminate between mild-moderate dementia. When 75 was used as the threshold between moderate-severe dementia, the sensitivity and the specificity were 73.9 and $67.7 \%$, respectively.
\end{abstract}

Conclusions: The IQCODE is moderately effective for assessing the severity of cognitive impairment in patients with $A D$.

Keywords: IQCODE, Alzheimer's disease, Dementia

\section{Background}

Alzheimer's disease $(\mathrm{AD})$ is the most common form of neurodegenerative disease associated with dementia among the elderly [1]. AD is characterized by a progressive decline in cognitive function that often begins with early disturbances in episodic memory and ultimately leads to absolute functional impairment $[2,3]$. Currently, the severity of cognitive dysfunction in patients with $\mathrm{AD}$ is often assessed with various instruments, including the Clinical Dementia Rating

\footnotetext{
* Correspondence: liuyan151617@163.com

†Yunlong Ding and Jiali Niu contributed equally to this work.

'Department of Neurology, JingJiang People's Hospital, No. 28, Zhongzhou

Road, Jingjiang CN 214500, Jiangsu, China

Full list of author information is available at the end of the article
}

(CDR), the Mini-Mental State Examination (MMSE) and patient-administered assessment tools in clinical trials and clinical studies [4]. Unfortunately, the effectiveness of these assessments may be influenced by several factors such as age, gender, educational level, and premorbid intelligence of the patients [5-8].

The Informant Questionnaire on Cognitive Decline in the Elderly (IQCODE) uses collateral information to assess changes in everyday cognitive functions over the previous 10-year period [9]. The IQCODE is based on a structured interview in which responses of informants who are often the spouses or relatives of patients who know the patients well are collected [10]. As an informant questionnaire, the IQCODE asks a series of

(c) The Author(s). 2018 Open Access This article is distributed under the terms of the Creative Commons Attribution 4.0 International License (http://creativecommons.org/licenses/by/4.0/), which permits unrestricted use, distribution, and reproduction in any medium, provided you give appropriate credit to the original author(s) and the source, provide a link to the Creative Commons license, and indicate if changes were made. The Creative Commons Public Domain Dedication waiver (http://creativecommons.org/publicdomain/zero/1.0/) applies to the data made available in this article, unless otherwise stated. 
questions about how the patient's cognition and functioning have changed, and as a result, it is not affected by the patient's premorbid intelligence or education [8]. Currently, several studies have already shown that the IQCODE is widely effective as a complementary screening test for dementia and is used within various cultural contexts, including Australian, Spanish, Lebanese, and Chinese [9, 11-17]. In a study of a Singaporean population, for instance, the combination of cognitive testing and the IQCODE provided better detection of dementia when individuals had no education [18]. Additionally, a study using a Spanish sample found that the IQCODE was more sensitive for identifying mild dementia than the MMSE [17]. A short form of the IQCODE that contained 16 items was subsequently developed; this version was highly correlated with the original version and possessed comparable validity [16]. The IQCODE is widely used as a complementary screening tool for dementia. However, there are few studies that have evaluated the efficacy of the IQCODE for assessing the severity of cognitive impairments in patients with $\mathrm{AD}$.

Therefore, this study recruited $\mathrm{AD}$ patients and interviewed their informants with the 16-item IQCODE to explore its clinical value for assessing the severity of cognitive impairments in patients with $\mathrm{AD}$.

\section{Methods}

\section{Study participants}

A total of 394 participants who were admitted to Jingjiang People's Hospital (201 cases) and Huashan Hospital at Fudan University (193 cases) from January 2013 to August 2016 were included in the study. Among all the patients, there were 184 males and 210 females. The patients' ages ranged from 43 to 91 years, with a mean age of 70.01 years $(\mathrm{SD}=9.77)$, and their education levels varied from illiterate to bachelor's degrees. Following a series of examinations, including a medical history, a clinical examination, neuropsychological testing and laboratory assessments, all the patients' conditions met the criteria for "the diagnosis of dementia due to Alzheimer's disease" that was recommended by the National Institute on Ageing-Alzheimer's Association workgroups [19]. All the informants, including 128 spouses, 215 children and 51 other relatives, were individuals who had been living with the patients for more than 10 years, contacted them at least 4 times a week and knew the patients' information well. Informed consent was obtained from participants with the capacity to consent. If they did not have capacity, a guardian or close family member was asked to give agreement for the person's participation, and sign his/her agreement for this. The experiment was approved by the local ethics committee.

\section{Procedure}

Three hundred and ninety-four patients with $\mathrm{AD}$ were classified into the following three groups by two senior neurologic physicians according to the severity of dementia as assessed by the CDR scores [20]: mild $(\mathrm{CDR}=1.0$, $n=107)$, moderate $(\mathrm{CDR}=2.0, n=194)$ and severe $(\mathrm{CDR}=3.0, n=93)$ dementia groups. If the two physicians had different opinions on the grouping, a third senior neurologic physician intervened until an agreement was reached. Each patient was assessed by a geriatrician who administered the MMSE [21], the Mattis Dementia Rating Scale (DRS) [22], and the Alzheimer's Disease Assessment Scale-Cognitive Subscale (ADAS-Cog) [23]. In addition, the informants were interviewed with the short version of the IQCODE containing 16 items.

Cognitive functions that were tested with the short IQCODE included short- and long-term memory, space and time orientation, calculation, learning and administration. Each item was ranked on a 5-point scale with 1 representing "much improved", 2 representing "a bit improved", 3 representing "not much change", 4 representing "a bit worse" and 5 representing "much worse". The sum of the scores was averaged over the 16 items to yield a total score ranging from 1 to 5 , with higher scores representing greater impairment [16].

\section{Statistical analysis}

Statistical analysis was performed with SPSS version 17.0 software (SPSS, Chicago, IL, USA). The data are presented as the mean \pm standard deviation (SD). Agreement between the 2 independent physicians was determined using kappa statistics, and $\mathrm{k}$ values ranging from 0.8 to 1.0 and from 0.6 to 0.8 were considered to represent nearly perfect agreement and substantial agreement, respectively. Clinical characteristics were evaluated using $X^{2}$ tests. Statistical differences between subject groups were tested using Student's t-test for normally distributed variables or Fisher's exact test for dichotomous variables. A receiver operating characteristic (ROC) curve analysis was employed to compare the accuracy of the IQCODE for assessing the severity of cognitive impairment in patients with AD. The area under the curve (AUC) was used as a measure of the dichotomous screening ability of the test and for choosing an optimal cut-off point that provided the largest AUC. The clinical significance was evaluated by analysing the correlation of the IQCODE scores with the MMSE scores, the DRS scores and the ADAS-Cog scores. Values of $P$ less than 0.05 were considered statistically significant.

\section{Results}

\section{Inter-observer variability}

The agreement of two senior neurologic physicians' in judging the severity of dementia was excellent, with a $\mathrm{K}$ 
value $0.894 \quad(P<0.001)$. The third senior neurologic physician intervened when they disagreed on the group until an agreement was reached.

\section{Clinical characteristics}

The participant characteristics are summarized by each stage of dementia in Table 1. A total of 394 AD patients participated in this study, of which 107 (27.2\%) patients were in the mild group, 194 (49.2\%) patients were in the moderate group, and $93(23.6 \%)$ patients were in the severe group. No significant differences were observed owing to age and sex among these three groups. However, the education levels in the mild, moderate and severe dementia groups were $6.21( \pm 1.70), 5.60( \pm 1.87)$, and $5.39( \pm 1.99)$, respectively, indicating that education level was negatively correlated with the severity of dementia $(P=0.004)$. The differences among the MMSE, DRS, and ADAS-Cog scores were statistically significant.

\section{Correlation between the IQCODE scores and neuropsychological testing}

Our results showed that the correlation coefficients of the IQCODE scores of the mild and severe dementia groups with neuropsychological testing, including the MMSE, DRS, and ADAS-Cog scores, were not statistically significant. However, the correlation coefficients of the IQCODE scores of moderate dementia with these three neuropsychological tests, the MMSE, DRS, and ADAS-Cog, were statistically significantly different $(-0.409,-0.324$, and 0.370 , respectively). Furthermore, significant differences were observed in the total sample, and the correlation coefficients with the MMSE, DRS, and ADAS-Cog were $-0.528,-0.436$, and 0.477 , respectively (Table 2).

\section{Effectiveness of the IQCODE in assessing the severity of cognitive impairment}

In the primary analysis, dementia of different severities had significantly different IQCODE scores for items, except the third item. The total scores from the mild, moderate and severe groups were $63.38( \pm 9.49), 68.39$
Table 2 Correlation of IQCODE scores and neuropsychological testing

\begin{tabular}{llll}
\hline & MMSE & DRS & ADAS-cog \\
\hline Mild dementia & 0.009 & 0.115 & 0.137 \\
Moderate dementia & $-0.409^{* *}$ & $-0.324^{* *}$ & $0.370^{* *}$ \\
Sever dementia & -0.131 & -0.193 & 0.197 \\
All & $-0.528^{* *}$ & $-0.436^{* *}$ & $0.477^{* *}$ \\
\hline
\end{tabular}

Abbreviations: MMSE the mini-mental state examination, DRS the Mattis =dementia rating scale, $A D A S$-cog the Alzheimer's disease assessment scale-cognitive subscale ${ }^{*} P<0.05,{ }^{* *} P<0.01$

$( \pm 8.73)$ and $75.96( \pm 3.52)$, respectively, and were statistically significant (Table 3 ). For discriminating between mild-moderate dementia, the ROC curve analyses for the IQCODE showed an AUC of 0.666 (95\% confidence interval (CI), 0.601-0.732). With 65 used as the cut-off point, the sensitivity was $66.1 \%$, and the specificity was $59.8 \%$. The AUC under the ROC curve for the IQCODE discriminating between moderate and severe dementia was 0.768 (95\% CI, 0.715-0.822); the sensitivity was $73.9 \%$, and the specificity was $67.7 \%$ with 75 used as the cut-off score.

\section{Discussion}

The Informant Questionnaire is one of the methods used for the clinical assessment of cognitive impairments. Among various questionnaires, the IQCODE is one of the most widely used methods [12]. As the IQCODE is a reliable and validated informant-based instrument for assessing changes in everyday cognitive dysfunction over a 10-year period, it has been widely adopted by clinical researchers across different cultures and languages [8, 24]. In general, informant-based assessments do not rely on direct patient testing, thus making capturing changes over time possible and operable and making these assessments less prone to social-cultural biases [25, 26]. However, having an informant who has known the older individual in question for at least 10 years respond to the questions is a key requirement for the IQCODE [12]. The IQCODE has

Table 1 General materials of 394 cases (mean \pm sd)

\begin{tabular}{lllll}
\hline items & $\begin{array}{l}\text { Mild dementia } \\
(n=107)\end{array}$ & $\begin{array}{l}\text { Moderate dementia } \\
(n=194)\end{array}$ & $\begin{array}{l}\text { Severe dementia } \\
(n=93)\end{array}$ & $F(P)$ \\
\hline Age in years & $70.10 \pm 9.22$ & $70.57 \pm 10.05$ & $68.72 \pm 9.77$ & $1.137(0.322)$ \\
sex (male:female) & 52.55 & $90: 104$ & $42: 51$ & $0.124(0.883)$ \\
Educationg level & $6.21 \pm 1.70$ & $5.60 \pm 1.87$ & $5.39 \pm 1.99$ & $5.506(0.004)$ \\
MMSE & $19.62 \pm 2.04$ & $14.86 \pm 2.96$ & $8.29 \pm 3.25$ & $402.806(0.000)$ \\
DRS & $112.75 \pm 15.25$ & $88.80 \pm 13.16$ & $67.52 \pm 15.86$ & $236.362(0.000)$ \\
ADAS-cog & $29.10 \pm 11.08$ & $40.45 \pm 12.17$ & $53.27 \pm 9.38$ & $89.686(0.000)$ \\
\hline
\end{tabular}

Education level: 1.lliteracy, 2. Old-style private school or family education, 3.Adult literacy class or night school, 4. Primary school drop-outs, 5.Primary school, 6.junior high school, 7.senior high school, 8.College or above

Abbreviations: MMSE the mini-mental state examination, DRS the Mattis dementia rating scale, ADAS-cog the Alzheimer's disease assessment scale-cognitive subscale 
Table 3 Comparison of single item and total IQCODE score of different severity of dementia

\begin{tabular}{lllll}
\hline iterm & $\begin{array}{l}\text { Mild dementia } \\
(n=107)\end{array}$ & $\begin{array}{l}\text { Moderate dementia } \\
(n=194)\end{array}$ & $\begin{array}{l}\text { Severe dementia } \\
(n=93)\end{array}$ & $43)$ \\
\hline 1 & $4.07 \pm 0.72$ & $4.32 \pm 0.76^{* *}$ & $4.92 \pm 0.30^{* *}$ & $43.820(0.000)$ \\
2 & $4.58 \pm 0.80$ & $4.79 \pm 0.56^{*}$ & $4.94 \pm 0.25^{* *}$ & $9.474(0.000)$ \\
3 & $4.73 \pm 0.56$ & $4.81 \pm 0.52$ & $4.92 \pm 0.27^{*}$ & $3.763(0.024)$ \\
4 & $3.71 \pm 1.01$ & $4.34 \pm 0.92^{* *}$ & $4.71 \pm 0.72^{* *}$ & $32.130(0.000)$ \\
5 & $3.60 \pm 0.93$ & $3.51 \pm 0.79$ & $4.00 \pm 0.88^{* *}$ & $10.832(0.000)$ \\
6 & $3.74 \pm 0.92$ & $4.07 \pm 0.79^{* *}$ & $4.59 \pm 0.61^{* *}$ & $29.307(0.000)$ \\
7 & $3.92 \pm 0.84$ & $4.25 \pm 0.77^{* *}$ & $4.80 \pm 0.48^{* *}$ & $36.338(0.000)$ \\
8 & $3.75 \pm 0.89$ & $4.11 \pm 0.81^{* *}$ & $4.70 \pm 0.62^{* *}$ & $36.349(0.000)$ \\
9 & $3.86 \pm 0.92$ & $4.27 \pm 0.72^{* *}$ & $4.77 \pm 0.53^{* *}$ & $37.726(0.000)$ \\
10 & $3.94 \pm 0.88$ & $4.34 \pm 0.72^{* *}$ & $4.86 \pm 0.46^{* *}$ & $40.532(0.000)$ \\
11 & $4.59 \pm 0.75$ & $4.78 \pm 0.60^{*}$ & $4.91 \pm 0.32^{*}$ & $7.711(0.001)$ \\
12 & $3.62 \pm 0.78$ & $3.51 \pm 0.62^{* *}$ & $31.684(0.000)$ \\
13 & $3.71 \pm 0.88$ & $3.86 \pm 0.91^{*}$ & $3.75 \pm 0.60^{* *}$ & $39.380(0.000)$ \\
14 & $3.64 \pm 0.92$ & $4.08 \pm 0.91^{* *}$ & $4.75 \pm 0.54^{* *}$ & $42.858(0.000)$ \\
15 & $3.79 \pm 0.87$ & $4.08 \pm 0.92^{* *}$ & $4.80 \pm 0.47^{* *}$ & $4.82 \pm 0.55^{* *}$ \\
16 & $4.17 \pm 0.57$ & $4.10 \pm 0.89^{* *}$ & $75.96 \pm 3.52^{* *}$ & $25.291(0.000)$ \\
Total score & $63.38 \pm 9.49$ & $4.40 \pm 0.73^{* *}$ & $60.627(0.000)$ \\
\hline
\end{tabular}

Comparison of mild and moderate groups marked on moderate group, and that of moderate and severe groups marked on severe group, ${ }^{*} P<0.05 ;{ }^{* *} P<0.01$

performed at least as well as cognitive screening tests, such as the MMSE. Furthermore, an important advantage of the IQCODE over the MMSE lies in that it is not affected by the patient's premorbid intelligence or education [27]. These properties are important for obtaining acceptable diagnostic accuracy, which is required for screening tools [28]. Moreover, from a clinician's perspective, the strengths of the IQCODE are that it is copyright free, available in different languages, and relatively easy to complete. From the original version with 26 questions that was developed in 1989, the short 16-item IQCODE version was developed in 1994, and its performance was essentially identical to that of the original version [16, 29]. There have been many studies supporting the validity of the IQCODE for dementia screening, with a sensitivity ranging from 79 to $100 \%$ and a specificity ranging from 68 to $100 \%[16,30,31]$. However, no studies evaluating the efficacy of IQCODE for assessing the severity of cognitive impairments in patients with AD have been published.

This study was based on the 16-item version, and the cognitive changes were scored on a 5-point scale. Higher scores corresponded to greater cognitive decline. The ratings were averaged, composing a mean score between 1 and 5. The severity of cognitive impairments of the total of $394 \mathrm{AD}$ patients was assessed by the CDR and the short IQCODE and was tested by the MMSE, DRS and ADAS-Cog. These patients were classified into mild, moderate and severe dementia groups according to the severity of dementia. Our results demonstrated that in the moderate group, there was a statistically significant association between the IQCODE scores and the MMSE, DRS and ADAS-Cog scores. However, no statistical significance was observed in the mild and severe groups. These findings suggest that IQCODE is more accurate and efficient for assessing moderate dementia than for assessing mild or severe dementia.

Significant difference in each item in the 16-item IQCODE were found in the mild and moderate groups but not in the severe group. ROC curve analyses for the IQCODE discriminating between mild-moderate dementia showed an AUC of 0.666 . The sensitivity was $66.1 \%$, and the specificity was $59.8 \%$ with 65 used as the cut-off point. The AUC under the ROC curve for the IQCODE discriminating between moderate-severe dementia was 0.768 ; the sensitivity was $73.9 \%$, and the specificity was $67.7 \%$ when using 75 as the cut-off score. Our results revealed that the sensitivity and specificity of the IQCODE were not high enough. Hence, we proposed that there might be a relatively large deviation when using the IQCODE alone to assess the severity of cognitive impairments. Furthermore, the IQCODE is unsatisfactory for evaluating conditions or treatment effects of patients with AD. Collectively, we do not advocate the use of the IQCODE alone for assessing treatment efficacy. A combination of the IQCODE with objective cognitive function tests and evaluation of patients' behavioural and psychiatric symptoms is required to provide the basis for further treatment. 


\section{Conclusions}

In conclusion, with the application of the IQCODE to survey 394 patients, we believe that the IQCODE is moderately effective for assessing the severity of cognitive impairments in AD patients. However, it is recommended that other clinical dementia rating scales be used to supplement the IQCODE in order to increase the sensitivity and the specificity and avoid misjudgements.

\section{Abbreviations}

AD: Alzheimer's disease; ADAS-Cog: Alzheimer's Disease Assessment ScaleCognitive Subscale; AUC: the area under the curve; CDR: Clinical Dementia Rating; DRS: Mattis Dementia Rating Scale; IQCODE: Informant Questionnaire on Cognitive Decline in the Elderly; MMSE: Mini-Mental State Examination; ROC: Receiver operating characteristic

\section{Funding}

This work was funded by the Guidance plan for social development of Taizhou Municipal Science and Technology (ssf20160141). The funder had no role in the study design, data collection, data analysis, data interpretation, writing of the report, decision to publish, or preparation of the manuscript.

\section{Availability of data and materials}

The datasets analysed during the current study are available from the corresponding author on reasonable request.

\section{Authors' contributions}

$Y L$ and $Y L D$ conceived and designed the study. YL, YRZ, WPL, YLD, YZ and $\mathrm{CW}$ assessed and diagnosed the patients. All authors were involved in the acquisition, analysis and interpretation of the data, with JLN taking the primary role in statistical analysis. YLD and JLN drafted the manuscript. All authors were involved in revising the manuscript critically and gave final approval of the manuscript.

\section{Ethics approval and consent to participate}

The study was approved by the ethics review committee of Huashan Hospital at Fudan University and the medical ethics committee of Jingjiang People's Hospital. Informed consent was obtained from participants with the capacity to consent. If they did not have capacity, a guardian or close family member was asked to give agreement for the person's participation, and sign his/her agreement for this. Written consent was obtained from all participants.

\section{Consent for publication}

Not applicable.

\section{Competing interests}

The authors declare that they have no competing interests.

\section{Publisher's Note}

Springer Nature remains neutral with regard to jurisdictional claims in published maps and institutional affiliations.

\section{Author details \\ 'Department of Neurology, Jing Jiang People's Hospital, No. 28, Zhongzhou Road, Jingjiang CN 214500, Jiangsu, China. 'Department of Clinical Pharmacy, JingJiang People's Hospital, No. 28, Zhongzhou Road, Jingjiang CN 214500, Jiangsu, China. ${ }^{3}$ Department of Neurology, Huashan Hospital at Fudan University, No. 12, Urumqi Road, Shanghai 200040, CN, China.}

Received: 6 January 2018 Accepted: 13 June 2018

Published online: 19 June 2018

\section{References}

1. Grimm A, Friedland K, Eckert A. Mitochondrial dysfunction: the missing link between aging and sporadic Alzheimer's disease. Biogerontology. 2016; 17(2):281-96.
2. Yan R, Vassar R. Targeting the beta secretase BACE1 for Alzheimer's disease therapy. The Lancet Neurology. 2014;13(3):319-29.

3. Lyketsos CG, Targum SD, Pendergrass JC, Lozano AM. Deep brain stimulation: a novel strategy for treating Alzheimer's disease. Innovations in clinical neuroscience. 2012;9(11-12):10-7.

4. Balsis S, Benge JF, Lowe DA, Geraci L, Doody RS. How do scores on the ADAS-cog, MMSE, and CDR-SOB correspond? Clin Neuropsychol. 2015;29(7): 1002-9.

5. Morgado J, Rocha CS, Maruta C, Guerreiro M, Martins IP. Cut-off scores in MMSE: a moving target? Eur J Neurol. 2010;17(5):692-5.

6. Rowland JT, Basic D, Storey JE, Conforti DA. The Rowland universal dementia assessment scale (RUDAS) and the Folstein MMSE in a multicultural cohort of elderly persons. Int Psychogeriatr. 2006;18(1): 111-20.

7. Turner TH, Hinson V. Mattis dementia rating scale cutoffs are inadequate for detecting dementia in Parkinson's disease. Applied neuropsychology Adult. 2013;20(1):61-5

8. Park MH. Informant questionnaire on cognitive decline in the elderly (IQCODE) for classifying cognitive dysfunction as cognitively normal, mild cognitive impairment, and dementia. Int Psychogeriatr. 2017;29(9): 1461-7.

9. Fuh JL, Teng EL, Lin KN, Larson EB, Wang SJ, Liu CY, Chou P, Kuo BI, Liu HC. The informant questionnaire on cognitive decline in the elderly (IQCODE) as a screening tool for dementia for a predominantly illiterate Chinese population. Neurology. 1995:45(1):92-6.

10. Harrison JK, Stott DJ, McShane R, Noel-Storr AH, Swann-Price RS, Quinn TJ. Informant questionnaire on cognitive decline in the elderly (IQCODE) for the early diagnosis of dementia across a variety of healthcare settings. The Cochrane database of systematic reviews. 2016;11:CD011333.

11. Sanchez MA, Lourenco RA. Screening for dementia: Brazilian version of the informant questionnaire on cognitive decline on the elderly and its psychometric properties. Geriatr Gerontol Int. 2013:13(3):687-93.

12. Jorm AF. The informant questionnaire on cognitive decline in the elderly (IQCODE): a review. Int Psychogeriatr. 2004;16(3):275-93.

13. Lourenco RA, Sanchez MA. Accuracy of the Brazilian version of the informant questionnaire on cognitive decline in the elderly at screening for dementia in community-dwelling elderly participants: findings from FIBRARJ study. J Geriatr Psychiatry Neurol. 2014;27(3):212-9.

14. Quinn TJ, Fearon P, Noel-Storr AH, Young C, McShane R, Stott DJ. Informant questionnaire on cognitive decline in the elderly (IOCODE) for the diagnosis of dementia within community dwelling populations. The Cochrane database of systematic reviews. 2014;4:CD010079.

15. Bou-Orm IR, Khamis AM, Chaaya M. Determinants of poor cognitive function using A-IQCODE among Lebanese older adults: a cross-sectional study. Aging Ment Health. 2018;22(6):844-8

16. Phung TK, Chaaya M, Asmar K, Atweh S, Ghusn H, Khoury RM, Prince M Waldemar G. Performance of the 16-item informant questionnaire on cognitive decline for the elderly (IQCODE) in an Arabic-speaking older population. Dement Geriatr Cogn Disord. 2015:40(5-6):276-89.

17. Potter GG, Plassman BL, Burke JR, Kabeto MU, Langa KM, Llewellyn DJ, Rogers MA, Steffens DC. Cognitive performance and informant reports in the diagnosis of cognitive impairment and dementia in African Americans and whites. Alzheimer's \& dementia : the journal of the Alzheimer's Association. 2009;5(6):445-53.

18. Narasimhalu K, Lee J, Auchus AP, Chen CP. Improving detection of dementia in Asian patients with low education: combining the mini-mental state examination and the informant questionnaire on cognitive decline in the elderly. Dement Geriatr Cogn Disord. 2008;25(1):17-22.

19. McKhann GM, Knopman DS, Chertkow H, Hyman BT, Jack CR, Jr., Kawas CH, Klunk WE, Koroshetz WJ, Manly JJ, Mayeux R et al: The diagnosis of dementia due to Alzheimer's disease: recommendations from the National Institute on Aging-Alzheimer's Association workgroups on diagnostic guidelines for Alzheimer's disease. Alzheimer's \& dementia : the journal of the Alzheimer's Association 2011, 7(3):263-269.

20. Ousset PJ, Andrieu S, Reynish E, Puel M, Vellas B: Clinical evaluation of dementia in a cohort of 358 patients with the French version of the Clinical Dementia Rating (CDR) scale. La Revue de medecine interne 2003, 24 Suppl 3:283s-287s.

21. Folstein MF, Folstein SE, McHugh PR. "Mini-mental state". A practical method for grading the cognitive state of patients for the clinician. J Psychiatr Res. 1975;12(3):189-98. 
22. Chan AS, Choi MK, Salmon DP. The effects of age, education, and gender on the Mattis dementia rating scale performance of elderly Chinese and American individuals. J Gerontol Ser B Psychol Sci Soc Sci. 2001;56(6):P356-63.

23. Rosen WG, Mohs RC, Davis KL. A new rating scale for Alzheimer's disease. Am J Psychiatry. 1984;141(11):1356-64.

24. Blennow Nordstrom E, Lilja G, Arestedt K, Friberg H, Nielsen N, Vestberg S, Cronberg T. Validity of the IQCODE-CA: an informant questionnaire on cognitive decline modified for a cardiac arrest population. Resuscitation. 2017;118:8-14.

25. Jorm AF, Christensen $H$, Korten AE, Jacomb PA, Henderson AS. Informant ratings of cognitive decline in old age: validation against change on cognitive tests over 7 to 8 years. Psychol Med. 2000;30(4):981-5.

26. Harrison JK, Fearon P, Noel-Storr AH, McShane R, Stott DJ, Quinn TJ. Informant questionnaire on cognitive decline in the elderly (IQCODE) for the diagnosis of dementia within a general practice (primary care) setting. The Cochrane database of systematic reviews. 2014;7:CD010771.

27. Jorm AF, Scott R, Cullen JS, MacKinnon AJ. Performance of the informant questionnaire on cognitive decline in the elderly (IQCODE) as a screening test for dementia. Psychol Med. 1991;21(3):785-90.

28. Bossuyt PM, Reitsma JB, Bruns DE, Gatsonis CA, Glasziou PP, Irwig L, Lijmer JG, Moher D, Rennie D, de Vet HC, et al. STARD 2015: an updated list of essential items for reporting diagnostic accuracy studies. BMJ (Clinical research ed). 2015; h5527:351.

29. Jorm AF. A short form of the informant questionnaire on cognitive decline in the elderly (IQCODE): development and cross-validation. Psychol Med. 1994:24(1):145-53.

30. Perroco TR, Bustamante SE, Moreno Mdel P, Hototian SR, Lopes MA, Azevedo D, Litvoc J, Filho WJ, Bottino CM. Performance of Brazilian long and short IQCODE on the screening of dementia in elderly people with low education. Int Psychogeriatr. 2009;21(3):531-8.

31. Harwood DM, Hope T, Jacoby R. Cognitive impairment in medical inpatients. I: screening for dementia-is history better than mental state? Age Ageing. 1997;26(1):31-5

\section{Ready to submit your research? Choose BMC and benefit from}

- fast, convenient online submission

- thorough peer review by experienced researchers in your field

- rapid publication on acceptance

- support for research data, including large and complex data types

- gold Open Access which fosters wider collaboration and increased citations - maximum visibility for your research: over $100 \mathrm{M}$ website views per year 